\title{
Positive periodic solutions of second-order nonlinear neutral differential equations with variable coefficients
}

\author{
Hocine Gabsi, Abdelouaheb Ardjouni*, Ahcene Djoudi
}

\begin{abstract}
In this paper, we use Krasnoselskii's fixed point theorem to establish the existence of positive periodic solutions of second-order nonlinear neutral differential equations. Our techniques can be used and applied to study other classes of problems and extension some results.
\end{abstract}

\section{INTRODUCTION}

Neutral functional differential equations are not only an extension of ordinary delay differential equations but also provide good models in many fields including Biology, Mechanics and Economics. For example (see [4], [11], [18]) population dynamics, since a growing population consumes more (or less) food than a matured one, depending on individual species, this leads to neutral functional equations. Periodicity and stability of solutions of differential and difference equations have been studied extensively in recent times. We refer to the references (see [1]-[15], [17] and [18]) and references therein for a wealth of information on this subject. In this paper, we study the existence of a positive periodic solution of the neutral differential equation. The study on neutral functional differential equations is more intricate than ordinary delay differential equations, that is why comparing plenty of results on the existence of periodic solutions for various types of first-order or second-order ordinary delay differential equations, studies on periodic solutions for neutral differential equations are relatively less, and most of them are confined to first-order neutral differential equations (see [4], [6], [7], [8], [9], [12]-[14], [18]). Very recently, in [18], Wu and Wang discussed the second order neutral delay differential equation

$$
(x(t)-C x(t-\delta))^{\prime \prime}+a(t) x(t)=\lambda b(t) f(x(t-\tau(t))),
$$

where $\lambda$ is a positive parameter, $\delta$ and $C$ are constants with $|C|<1$.

2010 Mathematics Subject Classification. Primary: 34K20, 34K30; Secondary: 47H10.

Key words and phrases. Fixed points, neutral differential equations, positive periodic solutions.

Full paper. Received 1 December 2017, revised 15 July 2018, accepted 16 November 2018, available online 15 December 2018. 
In [8], existence of positive periodic solutions of

$$
(x(t)-C x(t-\tau(t)))^{\prime \prime}=a(t) x(t)+f(t, x(t-\tau(t)))
$$

and

$$
(x(t)-C x(t-\tau(t)))^{\prime \prime}=-a(t) x(t)-f(t, x(t-\tau(t))),
$$

where $\tau \in C\left(\mathbb{R}, \mathbb{R}^{+}\right), a \in C(\mathbb{R},(0, \infty)), f \in C\left(\mathbb{R}, \mathbb{R}^{+} \times \mathbb{R}^{+}\right)$and $a$, are $\tau \omega$ periodic functions and $f$ is $\omega$-periodic in the first variable, were investigated with $|C|<1$.

In this article, we investigate the existence of positive periodic solutions for the following two types of second-order neutral functional differential equations with mixed-delays

$$
(x(t)-C(t) x(t-\delta(t)))^{\prime \prime}=a(t) x(t)+f(t, x(t-\tau(t)))
$$

and

$$
(x(t)-C(t) x(t-\delta(t)))^{\prime \prime}=-a(t) x(t)-f(t, x(t-\tau(t))),
$$

where

(H1) $a: \mathbb{R} \rightarrow \mathbb{R}^{+}, f: \mathbb{R} \times \mathbb{R} \rightarrow \mathbb{R}$ and for an arbitrary $C: \mathbb{R} \rightarrow \mathbb{R}$, Also we as sum that $a, C, \delta, \tau$ are $\omega$-periodic functions and $f(t+\omega, x)=$ $f(t, x)$.

We offer existence criteria for the positive periodic solutions of (1) and (2). The interest of the paper the case when $|C(t)|>1$. So to circumvent the difficulties we choose conditions for the delay $\delta$ and $f$ so that (1) and (2) has positive periodic solutions.

Special cases of (1) and (2) have been considered and investigated by many authors. For example, Cheung, Ren and Han in [8], using the main tool employed Krasnoselskii's fixed point theorem dealing with a sum of two mappings, one is a contraction and the other is completely continuous. The same thing, in this paper, we obtain various sufficient conditions for the existence of positive periodic solutions of (1) and (2) by employing two available operator and applying Krasnoselskii's fixed point theorem. The main features of this exposition are the following. In section 2 , we introduce some notations, and state some preliminary results needed in later sections, then we give the Green's function of (1) and (2), which plays an important role in this paper. Also, we present the inversions of (1) and (2), and Krasnoselskii's fixed point theorem. For details on Krasnoselskii's theorem we refer the reader to [16]. In the last sections, we present our main results on existence of positive periodic solutions of (1) and (2). We our work extends and improves previous results in the literature such as, Cheung, Ren and Han [8]. 


\section{Preliminaries}

To describe the main result we use the following notations. For $\omega>0$, let $C_{\omega}$ be the set of all continuous scalar functions $x$, periodic in $t$ of period $\omega$. Then $\left(C_{\omega},\|\cdot\|\right)$ is a Banach space with the supremum norm

$$
\|x\|=\sup _{t \in \mathbb{R}}|x(t)|=\sup _{t \in[0, \omega]}|x(t)|,
$$

define

$$
C_{\omega}^{+}=\left\{x \in C_{\omega} \mid x>0\right\}
$$

and

$$
C_{\omega}^{-}=\left\{x \in C_{\omega} \mid x<0\right\} .
$$

In the following we denote by

$$
M=\sup \{a(t) \mid t \in[0, \omega]\}, \quad m=\inf \{a(t) \mid t \in[0, \omega]\}, \beta=\sqrt{M} .
$$

Theorem 2.1 ([16]). Let $\mathcal{M}$ be a closed bounded convex nonempty subset of a Banach space $(X,\|\cdot\|)$. Suppose that $\mathcal{A}$ and $\mathcal{B}$ map $\mathcal{M}$ into $\mathcal{M}$ such that

(i) $x, y \in \mathcal{M}$, implies $\mathcal{A} x+\mathcal{B} y \in \mathcal{M}$,

(ii) $\mathcal{A}$ is compact and continuous,

(iii) $\mathcal{B}$ is a contraction mapping.

Then there exists $x \in \mathcal{M}$ with $x=\mathcal{A} x+\mathcal{B} x$.

Then there exists $x \in \mathcal{M}$ with $x=\mathcal{A} x+\mathcal{B} x$.

To apply Theorem 2.1, we need to define a Banach space $X$, a closed convex subset $\Omega$ of $X$ and construct a mapping is a completely continuous. So, we let $(X,\|\cdot\|)=\left(C_{\omega},\|\cdot\|\right)$ and $\Omega=\left\{\varphi \in C_{\omega}: l \leq \varphi \leq L\right\}$, where $L$ is non-negative constant and $l$ is positive constant. In this section we obtain the existence of a positive periodic solution of (1) by considering the two cases primary $|C(t)|>1$ and secondary $|C(t)|<1$. We begin with $|C(t)|>1$. In order to obtain the $\omega$-periodic solution of (2), we define the function $F: \mathbb{R} \times C_{\omega} \rightarrow \mathbb{R}$ by

$$
F(t, x):=f(t, x(t-\tau(t)))+a(t) C(t) x(t-\delta(t)) .
$$

In such case we require that a some hypotheses.

(H2) The function $\sigma(t)=t-\delta(t)$ is strictly increasing and $r(\cdot)$ is its inverse.

(H3) We assume that $F: \mathbb{R} \times C_{\omega} \rightarrow \mathbb{R}^{+}$is a positive continuous function on $\mathbb{R} \times C_{\omega}$ that is

$$
F(t, x) \geq 0, \text { for all } x \in C_{\omega} \text { and } t \in \mathbb{R} .
$$

(H4) Suppose $F: \mathbb{R} \times C_{\omega} \rightarrow \mathbb{R}^{-}$is a negative continuous function on $\mathbb{R} \times C_{\omega}$, that is

$$
F(t, x)<0 \text { for all } x \in C_{\omega} \text { and } t \in \mathbb{R} .
$$


Denote

$$
C_{0}:=\min _{t \in \mathbb{R}} \frac{1}{|C(r(t))|} \text { and } C_{1}:=\max _{t \in \mathbb{R}} \frac{1}{|C(r(t))|} .
$$

3. Existence of POSitive PeRIOdic SOlution FOR (1)

Similar to the results in [8] we have the following lemmas.

Lemma 3.1. The equation

$$
\frac{\mathrm{d}^{2} y}{\mathrm{~d} t^{2}}-M y(t)=h(t), \quad h \in C_{\omega}
$$

has a unique $\omega$-periodic solution

$$
y(t)=\left(T_{1} h\right)(t):=\int_{t}^{t+\omega} K_{1}(t, s)(-h(s)) \mathrm{d} s,
$$

where

$$
K_{1}(t, s)=\frac{\exp (-\beta(s-t))+\exp (\beta(s-t-\omega))}{2 \beta(1-\exp (-\beta \omega))}, \quad s \in[t, t+\omega] .
$$

Clearly, one can, by a change of variables, we have

$$
y(t)=y(t+\omega) .
$$

Lemma 3.2. $K_{1}(\cdot, \cdot)$ has the properties

1) $\int_{r(t)}^{r(t)+\omega} K_{1}(r(t), s) \mathrm{d} s=\frac{1}{M}$ for all $t \in[0, \omega], s \in[r(t), r(t)+\omega]$.

2) $0<\frac{\exp (-\beta \omega / 2)}{\beta(1-\exp (-\beta \omega))} \leq K_{1}(r(t), s) \leq \frac{1+\exp (-\beta \omega)}{2 \beta(1-\exp (-\beta \omega))}$.

3) $K_{1}(r(t+\omega), s+\omega)=K_{1}(r(t), s)$.

The following lemma is essential for our results on existence of periodic solution of (1).

Lemma 3.3. Assume that all hypotheses (H1) and (H2) hold true. If $x \in C_{\omega}$ then $x$ is a solution of equation (1) if and only if

$$
\begin{aligned}
x(t) & =\frac{1}{C(r(t))} x(r(t))+\frac{1}{C(r(t))}(I-A)^{-1} \\
& \times \int_{r(t)}^{r(t)+\omega} K_{1}(r(t), s)[a(s) C(s) x(s-\delta(s))+f(s, x(s-\tau(s)))] \mathrm{d} s,
\end{aligned}
$$

where

$$
(A y)(t)=\int_{t}^{t+\omega} K_{1}(t, s)[M-a(s)] y(s) \mathrm{d} s .
$$

Proof. Let $x \in C_{\omega}$ be a solution of (1) so (1) it can be rewritten as

$$
\begin{aligned}
& \frac{\mathrm{d}^{2}}{\mathrm{~d} t^{2}}(x(t)-C(t) x(t-\delta(t)))-M(x(t)-C(t) x(t-\delta(t))) \\
= & -M(x(t)-C(t) x(t-\delta(t)))+a(t) x(t)+f(t, x(t-\tau(t))) .
\end{aligned}
$$


Taking

$$
y(t)=x(t)-C(t) x(t-\delta(t)) .
$$

Then (4) is transformed into

$$
\begin{aligned}
& \frac{\mathrm{d}^{2}}{\mathrm{~d} t^{2}} y(t)-M y(t) \\
& =-M y(t)+a(t) y(t)+a(t) C(t) x(t-\delta(t))+f(t, x(t-\tau(t))) \\
& =(a(t) y(t)-M y(t))-a(t) y(t)+a(t) x(t)+f(t, x(t-\tau(t))) \\
& =-(M-a(t)) y(t)+a(t) C(t) x(t-\delta(t))+f(t, x(t-\tau(t))) \\
& =-(M-a(t)) y(t)+F(t, x(t)):=h(t) .
\end{aligned}
$$

Since $h \in C_{\omega}$ from Lemma 3.1, we have

$$
\begin{aligned}
y(t)= & \int_{t}^{t+\omega} K_{1}(t, s)(-h(s)) \mathrm{d} s \\
= & \int_{t}^{t+\omega} K_{1}(t, s)(M-a(s)) y(s) \mathrm{d} s \\
& +\int_{t}^{t+\omega} K_{1}(t, s)(-F(s, x(s))) \mathrm{d} s \\
= & (A y)(t)+\int_{t}^{t+\omega} K_{1}(t, s)(-F(s, x(s))) \mathrm{d} s \\
= & (A y)(t)+T_{1}(a(t) C(t) x(t-\delta(t))+f(t, x(t-\tau(t)))) .
\end{aligned}
$$

This yields

$$
(I-A) y(t)=T_{1}(a(t) C(t) x(t-\delta(t))+f(t, x(t-\tau(t)))) .
$$

Therefore, since $\|A\| \leq 1-\frac{m}{M}<1$ then

$$
y(t)=(I-A)^{-1} T_{1}(a(t) C(t) x(t-\delta(t))+f(t, x(t-\tau(t)))) .
$$

Obviously

$$
\begin{aligned}
x(t)= & C(t) x(t-\delta(t)) \\
& +(I-A)^{-1} T_{1}(a(t) C(t) x(t-\delta(t))+f(t, x(t-\tau(t)))) \\
= & C(t) x(r(t)) \\
& +(I-A)^{-1} T_{1}(a(t) C(t) x(t-\delta(t))+f(t, x(t-\tau(t)))) .
\end{aligned}
$$

In fact that $t=r(\sigma(t))$ and since $A$ is a linear operator, so $(I-A)^{-1}$ it is also a linear operator. This yields

$$
\begin{aligned}
x(r(t))= & C(r(t)) x(t) \\
- & (I-A)^{-1} \int_{r(t)}^{r(t)+\omega} K_{1}(r(t), s) \\
& \times(a(s) C(s) x(s-\delta(s))+f(s, x(s-\tau(s)))) \mathrm{d} s .
\end{aligned}
$$


Consequently,

$$
\begin{aligned}
x(t)= & \frac{1}{C(r(t))} x(r(t)) \\
& +\frac{1}{C(r(t))}(I-A)^{-1} \int_{r(t)}^{r(t)+\omega} K_{1}(r(t), s) \\
& \times(a(s) C(s) x(s-\delta(s))+f(s, x(s-\tau(s)))) \mathrm{d} s .
\end{aligned}
$$

Remark 3.1. It is not difficult to check that any function $x(t)$ that satisfies (3) is also a $\omega$-periodic.

- The case $C(t)>1$.

To simplify notations we define the operators $S, B_{1}, Q_{1}: C_{\omega} \rightarrow C_{\omega}$ by

$$
\begin{aligned}
(S x)(t) & :=\frac{1}{C(r(t))} x(r(t)) \\
\left(B_{1} x\right)(t) & :=\int_{r(t)}^{r(t)+\omega} K_{1}(r(t), s) F(s, x(s)) \mathrm{d} s,
\end{aligned}
$$

and

$$
\left(Q_{1} x\right)(t):=\frac{1}{C(r(t))}(I-A)^{-1}\left(B_{1} x\right)(t) .
$$

In view of (5), (6), and the above analysis, the existence of periodic solutions for (1) is equivalent to the existence of solutions for the operator equation

$$
S x+Q_{1} x=x .
$$

Lemma 3.4. Suppose that (H1) and (H2) hold. If $S$ is given by (5), then the operator $S: C_{\omega} \rightarrow C_{\omega}$ is a contraction.

Proof. For any $\phi, \varphi \in C_{\omega}$, it is easy to see that

$$
|(S \phi)(t)-(S \varphi)(t)|=\left|\frac{1}{C(r(t))}\right||\phi(r(t))-\varphi(r(t))| \leq \frac{1}{|C(r(t))|}\|\phi-\varphi\|,
$$

which implies that

$$
\|S \phi-S \varphi\| \leq C_{1}\|\phi-\varphi\| .
$$

Since $C_{1}<1$, then $S$ is a contraction operator.

Lemma 3.5. Suppose that (H1)-(H3) hold, then $Q_{1}$ is completely continuous and satisfies

$$
0<C_{0}\left(B_{1} x\right)(t) \leq\left(Q_{1} x\right)(t) \leq C_{1} \frac{M}{m}\left\|\left(B_{1} x\right)(t)\right\| \text { for } t \in \mathbb{R}, \quad x \in C_{\omega} .
$$

Proof. For $x \in C_{\omega}$. By Neumann expansion of $(I-A)^{-1} B_{1}$, we have

$$
\begin{aligned}
(I-A)^{-1} B_{1} & =\left[I+A+A^{2}+\cdots+A^{n}+\cdots\right] B_{1} \\
& =B_{1}+A B_{1}+A^{2} B_{1}+\cdots+A^{n} B_{1}+\cdots
\end{aligned}
$$


Moreover, since $C(r(t))>1$ and by condition (H3), having in mind (7), we get for all $t \in \mathbb{R}$, and $x \in C_{\omega}$

$$
\left(Q_{1} x\right)(t)=\frac{1}{C(r(t))}(I-A)^{-1}\left(B_{1} x\right)(t) \geq \frac{1}{C(r(t))}\left(B_{1} x\right)(t)>0 .
$$

On the other hand, recalling that $\|A\| \leq 1-\frac{m}{M}$ and by (H3), we see that for all $t \in \mathbb{R}$, and $x \in C_{\omega}$

$$
\begin{aligned}
\left(Q_{1} x\right)(t) & =\frac{1}{C(r(t))}(I-A)^{-1}\left(B_{1} x\right)(t) \\
& \leq C_{1}\left\|(I-A)^{-1}\right\|\left\|\left(B_{1} x\right)(t)\right\| \\
& \leq C_{1} \frac{1}{1-\|A\|}\left\|\left(B_{1} x\right)(t)\right\| \\
& \leq C_{1} \frac{1}{1-1+\frac{m}{M}}\left(B_{1} x\right)(t)=C_{1} \frac{M}{m}\left\|\left(B_{1} x\right)(t)\right\| .
\end{aligned}
$$

Consequently,

$$
0<C_{0}\left(B_{1} x\right)(t)<\left(Q_{1} x\right)(t) \leq C_{1} \frac{M}{m}\left\|\left(B_{1} x\right)(t)\right\|
$$

for all $t \in \mathbb{R}$, and $x \in C_{\omega}$.

Since $A$ and $B_{1}$ are completely continuous, so is $Q_{1}$.

Let

$$
\Omega:=\left\{\varphi \in C_{\omega}: l \leq \varphi \leq L\right\},
$$

where $L$ is non-negative constant and $K$ is positive constant and we assume that there exist positive constants $k_{1}$ and $k_{2}$ such that

$$
k_{1} \leq F(t, x) \leq k_{2} \text { for all } t \in \mathbb{R} \text { and } x \in \Omega .
$$

So we have the following Theorem.

Theorem 3.1. Under the hypotheses (H1)-(H3) and the condition (8). In addition suppose that

$$
l=\frac{C_{0}}{M} k_{1} \text { and } L=\frac{C_{1}}{m\left(1-C_{1}\right)} k_{2} .
$$

Then (1) has at least one positive $\omega$-periodic solution $x$, with $l \leq x \leq L$.

Proof. Let $\Omega:=\left\{\varphi \in C_{\omega}: l \leq \varphi \leq L\right\}$. It is obvious that $\Omega$ is a bounded closed convex set in $C_{\omega}$. Moreover, for any $\varphi, \phi \in \Omega$, it is easy to verify that $S_{1}+Q_{1}$ is continuous and $(S \varphi)(t+\omega)+\left(Q_{1} \varphi\right)(t+\omega)=(S \varphi)(t)+\left(Q_{1} \varphi\right)(t)$. Next, we claim that $S \varphi+Q_{1} \phi \in \Omega$ for all $\varphi, \phi \in \Omega$. Since $C(\cdot)>0$ and $0<k_{1} \leq F(t, x) \leq k_{2}$, then for any $\varphi, \phi \in \Omega$, in view of Lemmas 3.4, 3.5 and the condition (8) that

$$
(S \varphi)(t)+\left(Q_{1} \phi\right)(t) \leq \frac{1}{|C(r(t))|}\|\varphi\|+\frac{M}{m} C_{1}\left\|\left(B_{1} x\right)(t)\right\|
$$




$$
\begin{aligned}
& \leq C_{1} L+\frac{M}{m} C_{1} \int_{r(t)}^{r(t)+\omega}\left|K_{1}(r(t), s) F(s, \phi(s))\right| \mathrm{d} s \\
& \leq C_{1} L+C_{1} \frac{M}{m} \frac{k_{2}}{M} \\
& \leq C_{1} L+\frac{C_{1}}{m} \frac{m\left(1-C_{1}\right) L}{C_{1}}=L .
\end{aligned}
$$

On the other hand, by Lemmas 3.4, 3.5 and condition (8) we have

$$
\begin{aligned}
(S \varphi)(t)+\left(Q_{1} \phi\right)(t) & \geq\left(S_{1} \varphi\right)(t)+C_{0}\left(B_{1} \phi\right)(t) \\
& \geq\left(S_{1} \varphi\right)(t)+C_{0} \int_{r(t)}^{r(t)+\omega} K_{1}(r(t), s) F(s, \phi(s)) \mathrm{d} s \\
& \geq C_{0} l+C_{0} \int_{r(t)}^{r(t)+\omega} K_{1}(r(t), s) F(s, \phi(s)) \mathrm{d} s \\
& \geq C_{0} k_{1} \int_{r(t)}^{r(t)+\omega} K_{1}(r(t), s) \mathrm{d} s \geq C_{0} k_{1} \frac{1}{M} \\
& \geq C_{0} \frac{l M}{C_{0}} \frac{1}{M}=l .
\end{aligned}
$$

Thus $S \varphi+Q_{1} \phi \in \Omega$, that is, $\left(S+Q_{1}\right)(\Omega) \subset \Omega$. Clearly, all the hypotheses of the Theorem 2.1 are satisfied. Then, there exists a fixed point $x \in \Omega$ such that $S x+Q_{1} x=x$. By lemma (3.3) it is also positive $\omega$-periodic solutions for (1).

- The case $C(t)<-1$.

Note that the case $C(t)>1$ and the case $C(t)<-1$ differ only by a sign. So the treatment is the same as the first case by the small change of the hypothesis between (H3) and (H4). Then, we have the following result can be proved by a similar way.

Lemma 3.6. Suppose that (H1), (H2) and (H4) hold. Then,

(i) The operator $S: C_{\omega} \rightarrow C_{\omega}$ is a contraction.

(ii) $Q_{1}$ is completely continuous and satisfies

$$
0<C_{0}\left|\left(B_{1} x\right)(t)\right| \leq\left(Q_{1} x\right)(t) \leq C_{1} \frac{M}{m}\left\|\left(B_{1} x\right)(t)\right\|
$$

for $t \in \mathbb{R}, x \in C_{\omega}$.

Proof. We use (H4) recalling that $C(t)<-1$ on $\mathbb{R}$ and the fat that $A$ is a linear operator we get for $t \in \mathbb{R}, x \in C_{\omega}$.

$$
\begin{aligned}
& \frac{1}{C(r(t))}(I-A)^{-1}\left(B_{1} x\right)(t) \\
& =\frac{1}{C(r(t))}(I-A)^{-1} \int_{r(t)}^{r(t)+\omega} K_{1}(r(t), s) F(s, x(s)) \mathrm{d} s
\end{aligned}
$$




$$
\begin{aligned}
& =\frac{-1}{C(r(t))}(I-A)^{-1} \int_{r(t)}^{r(t)+\omega} K_{1}(r(t), s)[-F(s, x(s))] \mathrm{d} s \\
& =\left(\frac{-1}{C(r(t))}\right)(I-A)^{-1} \int_{r(t)}^{r(t)+\omega} K_{1}(r(t), s)[-F(s, x(s))] \mathrm{d} s \\
& \geq C_{0}\left(\int_{r(t)}^{r(t)+\omega} K_{1}(r(t), s)[-F(s, x(s))] \mathrm{d} s\right) \\
& =C_{0}\left|\int_{r(t)}^{r(t)+\omega} K_{1}(r(t), s) F(s, x(s)) \mathrm{d} s\right| \\
& \geq C_{0}\left|\left(B_{1} x\right)(t)\right| .
\end{aligned}
$$

Also we have

$$
\left(Q_{1} x\right)(t)=\frac{1}{C(r(t))}(I-A)^{-1}\left(B_{1} x\right)(t) \leq C_{1} \frac{M}{m}\left\|\left(B_{1} x\right)(t)\right\| .
$$

Hence, $0<C_{0}\left|\left(B_{1} x\right)(t)\right| \leq\left(Q_{1} x\right)(t) \leq C_{1} \frac{M}{m}\left\|\left(B_{1} x\right)(t)\right\|$ for $t \in \mathbb{R}, x \in$ $C_{\omega}$.

Let $\Omega:=\left\{\varphi \in C_{\omega}: l \leq \varphi \leq L\right\}$ where $L$ is non-negative constant and $l$ is positive constant and we assume that

$$
M \leq-F(t, x) \leq \frac{m}{C_{1}} \text { for all } t \in \mathbb{R}, \quad x \in \Omega .
$$

So we have the following Theorem.

Theorem 3.2. Under the hypotheses Lemma 3.6 and the condition (9). Suppose that

$$
l=0 \text { and } L=1 \text {. }
$$

Then (1) has at least one positive $\omega$-periodic solution $x$ with. $0<x \leq 1$.

Proof. Let $\Omega:=\left\{\varphi \in C_{\omega}: 0 \leq \varphi \leq 1\right\}$. It is obvious that $\Omega$ is a bounded closed convex set in $C_{\omega}$. Moreover, for any $\varphi, \phi \in \Omega$, it is easy to verify that $S+Q_{1}$ is continuous and $(S x)(t+\omega)+\left(Q_{1} x\right)(t+\omega)=(S x)(t)+\left(Q_{1} x\right)(t)$, that is, $\left(S+Q_{1}\right)(\Omega) \subset C_{\omega}$.

Next, we claim that $S \varphi+Q_{1} \phi \in \Omega$ for all $\varphi, \phi \in \Omega$. Since $0<M \leq$ $-F(t, x) \leq \frac{m}{C_{1}}$, then for any $\varphi, \phi \in \Omega$, by Lemma 3.6, we have

$$
\begin{aligned}
& (S \varphi)(t)+\left(Q_{1} \phi\right)(t)=\frac{1}{C(r(t))} \varphi(r(t)) \\
& +\frac{1}{C(r(t))}(I-A)^{-1} \int_{r(t)}^{r(t)+\omega} K_{1}(r(t), s) \\
& \quad \times[a(s) C(s) \phi(s-\delta(s))+f(s, \phi(s-\tau(s)))] \mathrm{d} s \\
& \leq \frac{1}{|C(r(t))|}\left\|(I-A)^{-1}\right\| \int_{r(t)}^{r(t)+\omega} K_{1}(r(t), s)|F(s, \phi(s))| \mathrm{d} s
\end{aligned}
$$




$$
\leq C_{1} \frac{M}{m} \frac{1}{M} \frac{m}{C_{1}}=1 .
$$

On the other hand, by Lemma 3.3 and condition (9)

$$
\begin{aligned}
& (S \varphi)(t)+\left(Q_{1} \phi\right)(t)=\frac{1}{C(r(t))} \varphi(r(t)) \\
& +\frac{1}{C(r(t))}(I-A)^{-1} \int_{r(t)}^{r(t)+\omega} K_{2}(r(t), s) F(s, \phi(s)) \mathrm{d} s \\
& =\frac{1}{C(r(t))}\left[\varphi(r(t))+(I-A)^{-1} \int_{r(t)}^{r(t)+\omega} K_{1}(r(t), s) F(s, \phi(s)) \mathrm{d} s\right] \\
& \geq \frac{-1}{C(r(t))}\left[\int_{r(t)}^{r(t)+\omega} K_{1}(r(t), s)(-F(s, \phi(s))) \mathrm{d} s-\varphi(r(t))\right] \\
& \geq \frac{-1}{C(r(t))}\left[\int_{r(t)}^{r(t)+\omega} K_{1}(r(t), s)[-F(s, \phi(s))] \mathrm{d} s-\varphi(r(t))\right] \\
& \geq \frac{-1}{C(r(t))}\left[\frac{1}{M} k_{1}-1\right]=0 .
\end{aligned}
$$

Thus $S \varphi+Q_{1} \phi \in \Omega$ for all $\varphi, \phi \in \Omega$. Clearly, all the hypotheses of the Theorem 2.1 are satisfied. Thus there exists a fixed point $x \in \Omega$ such that $S x+Q_{1} x=x$. By lemma (3.3) it is also positive $\omega$-periodic solutions for (1).

\section{Existence of Positive PERIOdic SOlution FOR (2)}

Note that the (1) and (2) differ only by a sign in the first equation. So the treatment is the same as the first case. So, the below procedure can be carried out to obtain the positive periodic solution of (2) by the small change of the hypothesis between (H3) and (H4) also Lemma 4.2 is obtained by replacing Lemma 3.1 by Lemma 4.1. Then, we have the following result can be proved by a similar way as in Section above.

- The Case $C(t)>1$.

Similar to the results in [8] we have the following lemmas

Lemma 4.1. The equation

$$
\frac{\mathrm{d}^{2} y}{\mathrm{~d} t^{2}}+M y(t)=h(t), h \in C_{\omega},
$$

has a unique $\omega$-periodic solution

$$
y(t)=\left(T_{2} h\right)(t):=\int_{t}^{t+\omega} K_{2}(t, s) h(s) \mathrm{d} s,
$$

where

$$
K_{2}(t, s)=\frac{\cos \beta\left(\frac{\omega}{2}+t-s\right)}{2 \beta \cos \frac{\beta \omega}{2}}, s \in[t, t+\omega] \text {. }
$$


Lemma 4.2. $K_{2}(.,$.$) has the properties$

1) $\int_{r(t)}^{r(t)+\omega} K_{2}(r(t), s) \mathrm{d} s=\frac{1}{M}$ for all $t \in[0, \omega], s \in[r(t), r(t)+\omega]$.

2) If $M<\left(\frac{\pi}{\omega}\right)^{2}$ then

$$
0<\frac{\cos (\beta \omega / 2)}{2 \beta \sin (\beta \omega / 2)} \leq K_{2}(r(t), s) \leq \frac{1}{2 \beta \sin (\omega \beta / 2)} .
$$

3) $K_{2}(r(t+\omega), s+\omega)=K_{2}(r(t), s)$.

The following lemma is essential for our results on existence of periodic solution of (2).

Lemma 4.3. Assume that all hypotheses (H1) and (H2) hold true. If $x \in C_{\omega}$ then $x$ is a solution of equation (2) if and only if

$$
\begin{aligned}
x(t)= & \frac{1}{C(r(t))} x(r(t)) \\
& +\frac{1}{C(r(t))}(I-P)^{-1} \int_{r(t)}^{r(t)+\omega} K_{2}(r(t), s) \\
& \times[a(s) C(s) x(s-\delta(s))+f(s, x(s-\tau(s)))] \mathrm{d} s,
\end{aligned}
$$

where

$$
(P y)(t)=\int_{t}^{t+\omega} K_{2}(t, s)[M-a(s)] y(s) \mathrm{d} s .
$$

Proof. From Lemma 4.1, and a similarly as in the prove of Lemma 3.3.

Define operators $S, B_{2}, Q_{2}: C_{\omega} \rightarrow C_{\omega}$ by

$$
\begin{aligned}
(S x)(t) & :=\frac{1}{C(r(t))} x(r(t)), \\
\left(B_{2} x\right)(t) & :=\int_{r(t)}^{r(t)+\omega} K_{2}(r(t), s) F(s, x(s)) \mathrm{d} s
\end{aligned}
$$

and

$$
\left(Q_{2} x\right)(t):=\frac{1}{C(r(t))}(I-P)^{-1}\left(B_{2} x\right)(t) .
$$

In view of $(10),(11)$, and the above analysis, the existence of periodic solutions for (2) is equivalent to the existence of solutions for the operator equation

$$
S x+Q_{2} x=x .
$$

Remark 4.1. Notice that $S$ in this section is defined exactly the same as that in (5). Hence Lemma 3.4 still holds true.

Lemma 4.4. Suppose that (H1), (H2) and (H3) hold.

(i) If $S$ is given by (10), then the operator $S: C_{\omega} \rightarrow C_{\omega}$ is a contraction. 
(ii) Under the properties of $K_{2}(.,$.$) in Lemma 4.2. Q_{2}$ is completely continuous and satisfies

$$
0<C_{0}\left(B_{2} x\right)(t) \leq\left(Q_{2} x\right)(t) \leq C_{1} \frac{M}{m}\left\|\left(B_{2} x\right)(t)\right\|,
$$

for all $t \in \mathbb{R}, x \in C_{\omega}$.

Let

$$
\Omega:=\left\{\varphi \in C_{\omega}: l \leq \varphi \leq L\right\},
$$

where $L$ is nonnegative constant and $l$ is positive constant. and we assume that there exist positive constants $k_{1}$ and $k_{2}$ such that

$$
k_{1} \leq F(t, x) \leq k_{2} \text { for all } t \in \mathbb{R} \text { and } x \in \Omega .
$$

So we have the following Theorem.

Theorem 4.1. If $M<\left(\frac{\pi}{\omega}\right)^{2}$ and (12) hold. Under the hypotheses of Lemma 4.4. In addition suppose that

$$
l=\frac{C_{0}}{M} k_{1} \text { and } L=\frac{C_{1}}{m\left(1-C_{1}\right)} k_{2} .
$$

Then (2) has at least one positive $\omega$-periodic solution $x$ with $l<x \leq L$.

- The case $C(t)<-1$.

Here we recall the hypothesis (H4) and we assume that (H1), (H2) and (H4) hold. It follows, we denote

$$
\Omega_{1}:=\left\{\varphi \in C_{\omega}: 0<\varphi \leq 1\right\} .
$$

Lemma 4.5. (i) If $S$ is given by (10), then the operator $S: C_{\omega} \rightarrow C_{\omega}$ is a contraction.

(ii) Under the properties of $K_{2}(.,$.$) in Lemma 4.2. Q_{2}$ is completely continuous and satisfies

$$
0<C_{0}\left|\left(B_{2} x\right)(t)\right| \leq\left(Q_{2} x\right)(t) \leq C_{1} \frac{M}{m}\left\|\left(B_{2} x\right)(t)\right\|,
$$

for all $t \in \mathbb{R}, x \in C_{\omega}$.

Suppose that

$$
M \leq-F(t, x) \leq \frac{m}{C_{1}} \text { for all } t \in \mathbb{R} \text { and } x \in C_{\omega} .
$$

Theorem 4.2. If $M<\left(\frac{\pi}{\omega}\right)^{2}$. Under the hypotheses of Lemma 4.5. If suppose (13) hold. In addition suppose that

$$
l=0 \text { and } L=1 .
$$

Then (2) has at least one positive $\omega$-periodic solution $x$ with $0<x \leq 1$.

Remark 4.2. Under the hypotheses (H1) and (H2). We assume that $|C(t)|>1$ and $M<\left(\frac{\pi}{\omega}\right)^{2}$. If either the hypotheses (H3), (8) and (12) or (H4), (9) and (13) hold true. Then, (1)-(2) has at least one Positive $\omega$-periodic solution $x \in \Omega \cap \Omega_{1}$. 
Remark 4.3. It is obvious that the theorems $3.1,4.2$ which remains valid even when by letting $\delta(t)$ and $C(t)$ which are constant thus, our work extends and improves previous results in the [8]. Nevertheless, we show that these new idea can be used to extend and improve [11] and [4].

We recall now the second case $|C(t)|<1$. Here we distinguish between two case the first if $c(t)$ is a constant so it is proved in [8] but the last case we can concluded by result as in [4] when the terms $\int_{-\infty}^{0} k(r) g(t, x(t+r)) \mathrm{d} r$ is replaced by $C(t) x(t-\delta(t))$ in [4]. That is this turns out to be a special case of the general problem in [4].

\section{Conclusion}

In this paper, we provided the existence of positive periodic solutions with sufficient conditions for second-order nonlinear neutral differential equations. The main tool of this paper is the method of fixed points. However, by introducing new fixed mappings, we get new existence conditions. The obtained results have a contribution to the related literature, and they improve and extend the results in Cheung, Ren and Han [8] from the cases of variable coefficient with $|C|<1$ to that variable coefficient cases with $|C(t)|<1$ and $|C(t)|>1$.

\section{REFERENCES}

[1] A. Ardjouni, A. Djoudi, Stability in Nonlinear Neutral Differential Equations with Infinite Delay, Mathematica Moravica, 18 (2) (2014), 91-103.

[2] A. Ardjouni, A. Djoudi, Stability for Nonlinear Neutral Integro-Differential Equations with Variable Delay, Mathematica Moravica, 19 (2) (2015), 1-18.

[3] A. Ardjouni, A. Djoudi, Periodic Solutions for Impulsive Neutral Dynamic Equations with Infinite Delay on Time Scales, Kragujevac Journal of Mathematics, 42 (1) (2018), 69-82.

[4] A. Ardjouni, A. Rezaiguia, A. Djoudi, Existence of positive periodic solutions for two types of second-order nonlinear neutral differential equations with infinite distributed delay, Journal of Applied Mathematics and Computing, 47 (1-2) (2015), 291-314.

[5] F. Bouchelaghem, A. Ardjouni, A. Djoudi, Positive Solutions for a Second-Order Difference Equation with Summation Boundary Conditions, Kragujevac Journal of Mathematics, 41 (2) (2017), 167-178.

[6] T. Candan, Existence of positive periodic solutions of first order neutral differential equations with variable coefficients, Appl Math Lett, 52 (2016), 142-148.

[7] T. Candan, Existence of positive periodic solutions of first order neutral differential equations, Math Methods Appl Sci, 40 (2017), 205-209.

[8] W. S. Cheung, J. Ren, W. Han, Positive periodic solutions of second order neutral functional differential equations, Nonlinear Analysis, 71 (2009), 3948-3955.

[9] M. Fan, K. Wang, Periodicity in a delayed ratio-dependent predator-prey system, J. Math. Anal. Appl., 262 (2001) 179-190. 
[10] K. Gopalsamy, M. R. S. Kulenović, G. Ladas, Environmental periodicity and time delays in a food-limited population model, Journal of Mathematical Analysis and Applications, 147 (2) (1990), 545-555.

[11] W. Han, J. Ren, Some results on second-order neutral functional differential equations with infinite distributed delay, Nonlinear Anal., 70 (2009), 1393-1406.

[12] H. F. Huo, W. T. Li, Positive periodic solutions of a class of delay differential system with feedback control, Applied Mathematics and Computation, 148 (2004), 35-46.

[13] H. F. Huo, W. T. Li, S. S. Cheng, Periodic solutions of two-species diffusion models with continuous time delays, Demonstratio Mathematica, XXXV (2) (2002), 432-446.

[14] Y. Kuang, Delay Differential Equations with Application in Population Dynamics, Academic Press, New York, 1993.

[15] M. B. Mesmouli, A. Ardjouni, A. Djoudi, Periodic Solutions for a System of Nonlinear Neutral Functional Difference Equations with Two Functional Delays, Mathematica Moravica, 19 (1) (2015), 57-71.

[16] D. R. Smart, Fixed point theorems, Cambridge Uni. Press., Cambridge, 1980.

[17] I. Soualhia, A. Ardjouni, A. Djoudi, Periodic solutions for neutral nonlinear difference equations with functional delay, Mathematica Moravica, 20 (1) (2016), 17-29.

[18] J. Wu, Z. C. Wang, Two periodic solutions of second-order neutral functional differential equations, Journal of Mathematical Analysis and Applications, 329 (2007), $677-689$.

\title{
Hocine Gabsi
}

Department of Mathematics

UNIVERSITY OF EL-OUED

EL-OUED

Algeria

E-mail address: hocinegabsi@gmail.com

\section{Abdelouaheb Ardjouni}

Department of MATHEMATICS AND InFormatics

University of Souk Ahras

P.O. Box 1553, Souk Ahras

Algeria

E-mail address: abd_ardjouni@yahoo.fr

\author{
Ahcene Duoudi \\ Department of Mathematics \\ UNIVERSITY OF ANNABA \\ P.O. Box 12, AnNaba \\ Algeria \\ E-mail address: adjoudi@yahoo.com
}

